\title{
MOMENTS OF THE INVERSE SCATTERING OPERATOR OF THE BOLTZMANN EQUATION: THEORY AND APPLICATIONS*
}

\author{
S. C. BRUGGER ${ }^{\dagger}$, A. SCHENK ${ }^{\dagger}$, AND W. FICHTNER ${ }^{\dagger}$
}

\begin{abstract}
In this paper useful physical objects called moments of the inverse scattering operator (MISO) of the Boltzmann equation (BE) are studied. The existence and uniqueness of the MISO is proven and a simple, generally valid, iterative scheme to actually compute those objects is given. The applications of the MISO extend from the computation of the solution for the space-homogeneous BE for small electric and magnetic fields to the exact computation of any transport parameter. This can be done for all moments of the space-inhomogeneous BE and for arbitrary electric and magnetic field intensities. The concept of MISO offers an elegant way to avoid the relaxation time approximation (RTA) every time it comes into play, not only theoretically but also in practical computations.
\end{abstract}

Key words. Boltzmann equation, transport model, beyond the relaxation time approximation, inverse scattering operator, semiconductor

AMS subject classifications. 82D $37,82 \mathrm{C} 70,82 \mathrm{C} 05,82 \mathrm{C} 80$

DOI. $10.1137 / 050633275$

1. Introduction. In contemporary semiconductor device simulation two approaches are common: For industrial application the so-called transport models (TMs) are widely used to compute terminal currents and to perform small signal and noise analysis. On the research side, one prefers, when possible, to directly solve the spaceinhomogeneous Boltzmann equation (BE) either using Monte Carlo (MC) methods or directly. The two methods often lead to different results. When trying to understand the origin of these discrepancies, one is immediately confronted with the relaxation time approximation (RTA). The RTA has been used to derive the TMs from the BE as a standard method mainly due to the lack of alternative ways to treat the problem. In the case of device simulation, it is impossible to validate the correctness of the RTA because there is no general method for directly comparing it with exact solutions.

The aim of this paper is to develop a method that avoids the RTA. It will allow, among others, the exact computation of transport parameters and noise sources for any moment of the BE using the MC method. The key feature in this formalism is the derivation and computation of moments of the inverse scattering operator (MISO). Although the formalism was primarily developed to be applied to semiconductors, it can also be used to analyze any open system described by a BE (linear or not).

The paper is organized in four sections. First, we recall the approximations in deriving a TM and point out how the knowledge of the MISO enables us to locally compare term by term the TM with the outcome of an MC simulation, i.e., with the solution of the BE. In section 3, we will outline the sufficient mathematical conditions under which the MISO exist, and we will present a natural way to compute them. In section 4, five useful applications are presented. First, the MISO are used to compute the solution of the space-homogeneous $\mathrm{BE}$ for small electric and magnetic fields. Then, transport parameters, Hall factors, and relaxation times (RTs) are computed in a very general way. At the end of the section, an exact expression is derived for

\footnotetext{
${ }^{*}$ Received by the editors June 8, 2005; accepted for publication (in revised form) January 9, 2006; published electronically March 24, 2006.

http://www.siam.org/journals/siap/66-4/63327.html

${ }^{\dagger}$ Institut für Integrierte Systeme, ETH Zürich, CH-8092 Zürich, Switzerland (brugger@iis.ee. ethz.ch, schenk@iis.ee.ethz.ch, fw@iis.ee.ethz.ch).
} 
the correlation functions of the Langevin noise sources for arbitrary moments of the space-homogeneous nondegenerate BE. Finally, section 5 gives conclusions and a brief outlook on future work.

\section{Direct comparison of TMs with the BE.}

2.1. Derivation of TMs from the BE. The first step in the derivation of any TM from the BE

$$
\partial_{t} f+\vec{v} \cdot \nabla_{\mathbf{r}} f-\frac{q}{\hbar} \vec{E} \cdot \nabla_{\mathbf{k}} f=S f
$$

is to introduce some kind of $\mathrm{RT} \tau$, which ideally should contain all information about the scattering operator $(\mathrm{SO}) S$ :

$$
\partial_{t} f+\vec{v} \cdot \nabla_{\mathbf{r}} f-\frac{q}{\hbar} \vec{E} \cdot \nabla_{\mathbf{k}} f=\frac{f-\frac{n}{n_{e q}} f_{e q}}{\tau(f, \vec{r}, t, \vec{k}, \vec{E})} .
$$

The function $\tau$ may depend on moments of the distribution function $f$, the position in real space $\vec{r}$, the momentum $\vec{k}$, the time $t$, and the electric field $\vec{E}$. Multiplying both sides of (2.2) by $\tau$ results in

$$
\begin{gathered}
\tau(f, \vec{r}, t, \vec{k}, \vec{E}) \partial_{t} f+\tau(f, \vec{r}, t, \vec{k}, \vec{E}) \vec{v} \cdot \nabla_{\mathbf{r}} f \\
-\tau(f, \vec{r}, t, \vec{k}, \vec{E}) \frac{q}{\hbar} \vec{E} \cdot \nabla_{\mathbf{k}} f=f-\frac{n}{n_{e q}} f_{e q} .
\end{gathered}
$$

In (2.1)-(2.3), $f_{e q}$ denotes the Boltzmann distribution function normalized to one $\left(\int_{B z} f_{e q}^{2}(\vec{k}) \mathrm{d}^{3} k=1\right)$ and $n:=\int_{B z} f(\vec{k}) \mathrm{d}^{3} k, n_{e q}:=\int_{B z} f_{e q}(\vec{k}) \mathrm{d}^{3} k$, with $B z$ the Brillouin zone. The symbol $n_{e q}$ is used here for convenience, although the actual equilibrium density differs from $n_{e q}$ by a constant due to the normalization condition.

To be able to compare (2.2) and (2.3) with the exact BE (2.1), one has to invert the SO (2.4):

$$
\begin{aligned}
\int S^{-1}\left(\vec{k}, \vec{k}^{\prime}\right) \partial_{t} f\left(\vec{k}^{\prime}\right) d^{3} k^{\prime}+\int S^{-1}\left(\vec{k}, \vec{k}^{\prime}\right) \vec{v}\left(\vec{k}^{\prime}\right) \cdot \nabla_{\mathbf{r}} f\left(\vec{k}^{\prime}\right) d^{3} k^{\prime} \\
-\int S^{-1}\left(\vec{k}, \vec{k}^{\prime}\right) \frac{q}{\hbar} \vec{E} \cdot \nabla_{\mathbf{k}^{\prime}} f\left(\vec{k}^{\prime}\right) d^{3} k^{\prime}=f(\vec{k})-\frac{n}{n_{e q}} f_{e q}(\vec{k}) .
\end{aligned}
$$

Note that $S^{-1} \circ S \neq \mathbb{1 1}$ ! This crucial statement will be explained in detail in section 3 .

RTs are introduced because the original BE (2.1) causes a major problem when one wants to derive a transport equation: The distribution function $f$ does not appear isolated in the equation. The only term in which $f$ is isolated is $\partial_{t} f$, which is the partial derivative of $f$ by the time, which is not an equation for $f$. By introducing an $\mathrm{RT} \tau, f$ appears isolated on the rhs of the equation. This is the main reason why RTs are introduced. To do the same in an exact way, the simplest possibility is to reverse the SO. This is the only possibility for expressing in an exact mathematical way what the RTA tries to achieve on a heuristic basis. In (2.2), the term $-\frac{n}{n_{e q}} f_{e q}$ was introduced phenomenologically to express the fact that at thermodynamic equilibrium the collision term disappears. The mathematical reason is, however, that the SO $S$ has an eigenvector with eigenvalue 0 , which is the equilibrium distribution $f_{e q}$ in the case of Boltzmann statistics. Therefore, (2.4) is the rigorous mathematical formulation of what was done approximately and heuristically by introducing an RT. 
The second step is to build a moment of interest of (2.3) by multiplying (2.3) by a function $g(\vec{k})$ and integrating over the momentum space,

$$
-\int_{B z}\left[g(\vec{k}) \tau(f(\vec{k}, \vec{r})) \frac{q}{\hbar} \vec{E} \cdot \nabla_{\mathbf{k}} f(\vec{k}, \vec{r})\right] \mathrm{d}^{3} k=\int_{B z} g(\vec{k})\left[f(\vec{k}, \vec{r})-\frac{n}{n_{e q}} f_{e q}(\vec{k}, \vec{r})\right] \mathrm{d}^{3} k,
$$

where $g(\vec{k})$ is a function in which we are interested. $g(\vec{k})$ could be, e.g., a power of the velocity.

Building moments in a similar fashion with (2.4) leads to

$$
\begin{gathered}
\int_{B z} \int_{B z}\left[g(\vec{k}) S^{-1}\left(\vec{k}, \vec{k}_{1}, \vec{r}\right) \partial_{t} f\left(\vec{k}_{1}, \vec{r}\right)\right] \mathrm{d}^{3} k_{1} \mathrm{~d}^{3} k \\
\quad+\int_{B z} \int_{B z}\left[g(\vec{k}) S^{-1}\left(\vec{k}, \vec{k}_{1}, \vec{r}\right) \vec{v}\left(\vec{k}_{1}\right) \cdot \nabla_{\mathbf{r}} f\left(\vec{k}_{1}, \vec{r}\right)\right] \mathrm{d}^{3} k_{1} \mathrm{~d}^{3} k \\
\quad-\int_{B z} \int_{B z}\left[g(\vec{k}) S^{-1}\left(\vec{k}, \vec{k}_{1}, \vec{r}\right) \frac{q}{\hbar} \vec{E} \cdot \nabla_{\mathbf{k}_{1}} f\left(\vec{k}_{1}, \vec{r}\right)\right] \mathrm{d}^{3} k_{1} \mathrm{~d}^{3} k \\
:=\int_{B z} S_{g}^{-1}\left(\vec{k}_{1}, \vec{r}\right) \partial_{t} f\left(\vec{k}_{1}, \vec{r}\right) \mathrm{d}^{3} k_{1}+\int_{B z} S_{g}^{-1}\left(\vec{k}_{1}, \vec{r}\right) \vec{v}\left(\vec{k}_{1}\right) \cdot \nabla_{\mathbf{r}} f\left(\vec{k}_{1}, \vec{r}\right) \mathrm{d}^{3} k_{1} \\
-\int_{B z} S_{g}^{-1}\left(\vec{k}_{1}, \vec{r}\right) \frac{q}{\hbar} \vec{E} \cdot \nabla_{\mathbf{k}_{1}} f\left(\vec{k}_{1}, \vec{r}\right) \mathrm{d}^{3} k_{1}=\int_{B z} g(\vec{k})\left[f(\vec{k}, \vec{r})-\frac{n}{n_{e q}} f_{e q}(\vec{k}, \vec{r})\right] \mathrm{d}^{3} k
\end{gathered}
$$

where

$$
S_{g}^{-1}\left(\vec{k}_{1}, \vec{r}\right)=\int_{B z} g(\vec{k}) S^{-1}\left(\vec{k}, \vec{k}_{1}, \vec{r}\right) \mathrm{d}^{3} k
$$

is a moment of the inverse scattering operator (ISO) in (2.5).

The third step is to perform a partial integration of the $\mathbf{k}$-gradient term in (2.5) and to neglect the boundary term

$$
\int_{B z} g(\vec{k}) \tau(f(\vec{k}, \vec{r})) \frac{q}{\hbar} \vec{E} \cdot \nabla_{\mathbf{k}} f(\vec{k}, \vec{r}) \mathrm{d}^{3} k \simeq-\frac{q}{\hbar} \vec{E} \cdot \int_{B z} \nabla_{\mathbf{k}}(g(\vec{k}) \tau(f(\vec{k}, \vec{r}))) f(\vec{k}, \vec{r}) \mathrm{d}^{3} k .
$$

Because the function $S_{g}^{-1}\left(\vec{k}_{1}, \vec{r}\right)$ can be discontinuous in some points (for some silicon MC models, this happens on the boundary surfaces between two valleys), we cannot exactly transform the $\mathbf{k}$-gradient term of (2.6) as in (2.8). Instead we have to decompose the domain $B z$ into subdomains, where the function $S_{g}^{-1}\left(\vec{k}_{1}, \vec{r}\right)$ is continuous, and keep all the boundary terms, which in general will not disappear, as follows:

$$
\begin{aligned}
& \sum_{i} \int_{B z_{i}} S_{g}^{-1}(\vec{k}, \vec{r}) \frac{q}{\hbar} \vec{E} \cdot \nabla_{\mathbf{k}} f(\vec{k}, \vec{r}) \mathrm{d}^{3} k \\
= & -\frac{q}{\hbar} \vec{E} \cdot \sum_{i} \int_{B z_{i}} \nabla_{\mathbf{k}}\left(S_{g}^{-1}(\vec{k}, \vec{r})\right) f(\vec{k}, \vec{r}) \mathrm{d}^{3} k+\frac{q}{\hbar} \vec{E} \cdot \sum_{i} \oint_{\partial B z_{i}} S_{g}^{-1}(\vec{k}, \vec{r}) f(\vec{k}, \vec{r}) \vec{n} \mathrm{da}
\end{aligned}
$$

where $\bigcup_{i} B z_{i}=B z$.

The boundary term $\sum_{i} \oint_{\partial B z_{i}} S_{g}^{-1}(\vec{k}, \vec{r}) f(\vec{k}, \vec{r}) \vec{n} \mathrm{~d} a$ does not vanish. To our knowledge it has never been investigated numerically whether this term is negligible for all 
moments and for all field strengths of practical interest, e.g., for bulk silicon. We will therefore keep this term in our numerical analysis.

To derive common TMs, only a small number of functions $g$ is relevant. For the drift-diffusion (DD) model, only the moment corresponding to $g_{1}=\vec{v}$ is needed. The hydrodynamic model is obtained by considering the moments $g_{1}=\vec{v}, g_{2}=\epsilon$ (where $\epsilon(\vec{k})$ is the energy) or $\|v\|^{2}$ and $g_{3}=\tau g_{2} \vec{v}$. Finally, for the so-called six moments method (see [6]), the additional moments $g_{4}=\epsilon^{2}$ and $g_{5}=\tau g_{4} \vec{v}$ are considered. These moments equations, together with the Poisson equation and the current continuity equation (the contraction of the BE with the constant function 1), constitute a TM that should approximate the solution to the BE coupled with the Poisson equation. These equations are usually parametrized using the electrostatic potential, the particle density(ies), and the mean value of $g_{2}$ and $g_{4}$. To close this system, further steps are needed: The $g_{3}$ (resp., $g_{5}$ ) moment is inserted into the $\nabla_{\mathbf{r}}$ term of the $g_{2}$ (resp., $g_{4}$ ) moment and approximations are done, such as the use of the Einstein relation, the replacement of tensorial transport coefficients by scalars, and the use of a closure relation for the last moment (see, e.g., [13], [3], [1], [12]). Finally, all expressions still unknown are called transport coefficients and computed using a model (analytical and/or bulk MC simulation) and parametrized by the functions listed above.

2.2. Transport parameters. In order to illustrate potential applications of the outlined concept, we directly compare the moments of well-known macroscopic TMs, such as the DD model and the energy-balance (EB) model (see, e.g., [12, Chap. 1.1.3]), with the corresponding terms from the BE containing the exact ISO. The equation for the current in the DD model reads

$$
\tau_{p} \partial_{t}(\langle\vec{v}\rangle)-\mu n \vec{E}-D \nabla_{\mathbf{r}} n=\langle\vec{v}\rangle=:-\frac{\vec{J}}{q},
$$

where $\tau_{p}$ is the momentum RT, $\mu$ the mobility tensor, $D$ the diffusivity tensor, $n$ the density, and $\langle\vec{v}\rangle:=\int_{B z} \vec{v} f \mathrm{~d}^{3} k(\langle\vec{v}\rangle / n$ is the mean velocity).

If we compare term by term the lhs of (2.10) with the lhs of (2.6), we find the following conditions for the DD model to be exact:

$$
\tau_{p} \partial_{t}\left(\left\langle v_{i}\right\rangle\right) \stackrel{!}{=} \int_{B z} S_{v_{i}}^{-1}\left(\vec{k}_{1}, \vec{r}\right) \partial_{t} f\left(\vec{k}_{1}, \vec{r}\right) \mathrm{d}^{3} k_{1}, \quad i=1, \ldots, 3,
$$

$$
\begin{array}{r}
(\mu n \vec{E})_{i} \stackrel{!}{=}-\frac{q}{\hbar} \vec{E} \cdot \int_{B z} \nabla_{\mathbf{k}}\left(S_{v_{i}}^{-1}(\vec{k}, \vec{r})\right) f(\vec{k}, \vec{r}) \mathrm{d}^{3} k+\frac{q}{\hbar} \vec{E} \cdot \sum_{j} \oint_{\partial B z_{j}} S_{v_{i}}^{-1}(\vec{k}, \vec{r}) f(\vec{k}, \vec{r}) \vec{n} \mathrm{~d} a, \\
i=1, \ldots, 3, \\
(2.13)\left(-D \nabla_{\mathbf{r}} n\right)_{i} \stackrel{!}{=} \int_{B z} S_{v_{i}}^{-1}\left(\vec{k}_{1}, \vec{r}\right) \vec{v}\left(\vec{k}_{1}\right) \cdot \nabla_{\mathbf{r}} f\left(\vec{k}_{1}, \vec{r}\right) \mathrm{d}^{3} k_{1}, \quad \mathrm{i}=1, \ldots, 3, \quad\left\langle v_{i}\right\rangle_{e q}=0 .
\end{array}
$$

Note that (2.13) gives a natural definition of the mobility tensor

$$
\begin{array}{r}
(\mu)_{i j}:=-\frac{q}{n \hbar} \int_{B z} \partial_{k_{j}}\left(S_{v_{i}}^{-1}(\vec{k}, \vec{r})\right) f(\vec{k}, \vec{r}) \mathrm{d}^{3} k+\frac{q}{n \hbar} \sum_{l} \oint_{\partial B z_{l}} S_{v_{i}}^{-1}(\vec{k}, \vec{r}) f(\vec{k}, \vec{r})(\vec{n} \mathrm{~d} a)_{j} \\
i=1, \ldots, 3 .
\end{array}
$$


The corresponding equations for the EB model are

$$
\tau_{p} \partial_{t}(\langle\vec{v}\rangle)-\mu n \vec{E}-\mu n \nabla_{\mathbf{r}}\left(\frac{k_{B}}{q} \frac{\langle T\rangle}{n}\right)-D^{\prime} \nabla_{\mathbf{r}} n=\langle\vec{v}\rangle,
$$

$$
\frac{3}{2} k_{B}(\langle T\rangle-n \underbrace{\frac{\langle T\rangle_{e q}}{n_{e q}}}_{:=T_{e q}})=-\tau_{E} q\langle\vec{v}\rangle \cdot \vec{E}-\tau_{E} \nabla_{\mathbf{r}}\left[-\kappa_{n} \nabla_{\mathbf{r}}\left(\frac{\langle T\rangle}{n}\right)+\frac{5 k_{B}}{2} \frac{\langle T\rangle\langle\vec{v}\rangle}{n}\right],
$$

where $\tau_{E}$ is the energy RT, $\kappa_{n}$ the heat conduction coefficient, $\langle T\rangle_{e q} / n_{e q}$ the temperature in thermodynamic equilibrium, $D^{\prime}:=\frac{k_{B}}{q} \frac{\langle T\rangle}{n} \mu$, and $\langle T\rangle:=\frac{m}{3 k_{B}} \cdot \operatorname{Tr}\left(\int_{B z}(\vec{v} \otimes \vec{v}) f \mathrm{~d}^{3} k\right)$. If we compare (2.15) and (2.16) with (2.6), we find the following conditions for the EB model to be exact:

$$
-\mu\left(\nabla_{\mathbf{r}}\left(\frac{k_{B}\langle T\rangle}{q}\right)\right)_{i} \stackrel{!}{=} \int_{B z} S_{v_{i}}^{-1}\left(\vec{k}_{1}, \vec{r}\right) \vec{v}\left(\vec{k}_{1}\right) \cdot \nabla_{\mathbf{r}} f\left(\vec{k}_{1}, \vec{r}\right) \mathrm{d}^{3} k_{1}, \quad i=1, \ldots, 3,
$$

$$
\begin{aligned}
\tau_{E} q\langle\vec{v}\rangle \cdot \vec{E} \stackrel{!}{=}-\frac{1}{2} m \sum_{i=1}^{3} \frac{q}{\hbar} \vec{E} \cdot & \int_{B z} \nabla_{\mathbf{k}}\left(S_{v_{i} v_{i}}^{-1}(\vec{k}, \vec{r})\right) f(\vec{k}, \vec{r}) \mathrm{d}^{3} k \\
& +\frac{1}{2} m \sum_{i=1}^{3} \frac{q}{\hbar} \vec{E} \cdot \sum_{j} \oint_{\partial B z_{j}} S_{v_{i} v_{i}}^{-1}(\vec{k}, \vec{r}) f(\vec{k}, \vec{r}) \vec{n} \mathrm{~d} a
\end{aligned}
$$

$$
-\tau_{E} \nabla_{\mathbf{r}}\left[-\kappa_{n} \nabla_{\mathbf{r}}\left(\frac{\langle T\rangle}{n}\right)+\frac{5 k_{B}}{2} \frac{\langle T\rangle\langle\vec{v}\rangle}{n}\right] \stackrel{!}{=} \frac{1}{2} m \sum_{i=1}^{3} \int_{B z} S_{v_{i} v_{i}}^{-1}\left(\vec{k}_{1}, \vec{r}\right) \vec{v}\left(\vec{k}_{1}\right) \cdot \nabla_{\mathbf{r}} f\left(\vec{k}_{1}, \vec{r}\right) \mathrm{d}^{3} k_{1} .
$$

Although the terms in the rhs of (2.11)-(2.13) and (2.17)-(2.19) look rather cumbersome, this is not the case. As soon as we know $S_{g}^{-1}(\vec{k}, \vec{r})$ for all required $g$, we can easily compute such terms for a given device with an MC simulation and then locally compare with the terms of the TM. It should be noted that by construction the following holds: If the transport coefficients computed by the MC method are reinserted into the TMs as a function of position only (not as a function of the density or the mean energy), then the TMs will exactly reproduce the MC density and the $\mathrm{MC}$ current density in the case of the DD and EB models, as well as the MC energy current density in the case of the EB model. Another way to verify this statement is to reinsert electric field, density, and mean energy from the MC solution of the $\mathrm{BE}$ into the TMs that contain the exact transport coefficients, and to observe that they indeed solve these equations. Therefore, the restrictions imposed on these TMs to be valid (restrictions arising from the models for the transport coefficients) become obsolete as soon as the exact expressions (2.11)-(2.13) and (2.17)-(2.19) are used.

Based on this motivation for the computation of MISO, we show how to actually compute them in a general way. 


\section{Existence and computation of the MISO.}

3.1. Derivation of an equation for the MISO. We start with the general form of the BE,

$$
\begin{aligned}
& (3.1) \quad \partial_{t} f(\vec{r}, t, \vec{k}, b)+\dot{\vec{r}} \cdot \nabla_{\mathbf{r}} f(\vec{r}, t, \vec{k}, b)+\dot{\vec{k}} \cdot \nabla_{\mathbf{k}} f(\vec{r}, t, \vec{k}, b) \\
& =\sum_{b_{0}} \int_{V_{b_{0}}} f\left(\vec{r}, t, \vec{k}_{0}, b_{0}\right) w(\vec{r}, t)\left(\vec{k}_{0}, b_{0} \mid \vec{k}, b\right) \mathrm{d}^{3} k_{0}-\sum_{b_{0}} \int_{V_{b_{0}}} f(\vec{r}, t, \vec{k}, b) w(\vec{r}, t)\left(\vec{k}, b \mid \vec{k}_{0}, b_{0}\right) \mathrm{d}^{3} k_{0},
\end{aligned}
$$

where $\vec{r}$ is the position in space, $\vec{k}$ the position in k-space, $b$ a band-valley index, $V_{b}$ the k-space of band-valley $b$, and $w(\vec{r}, t)\left(\vec{k}, b \mid \vec{k}_{0}, b_{0}\right)$ is the scattering rate from point $(\vec{k}, b)$ to $\left(\vec{k}_{0}, b_{0}\right)$ (at time $t$ and space position $\vec{r}$, respectively). Note that the Pauli blocking factors $(1-f)$ are included in the scattering rates $w$. Since $0<1-f \leq 1$, they will never increase the magnitude of $w$. This will be of some importance below. In the following we will work under the assumption that the $V_{b}$ are compact pairwise disjoint subsets of $\mathbb{R}^{3}$, and $w(\vec{r}, t)\left(\vec{k}, b \mid \vec{k}_{0}, b_{0}\right): V_{b} \times V_{b_{0}} \rightarrow \mathbb{R}$ is a continuous function of $\vec{k}$ and $\overrightarrow{k_{0}}$. We define $K:=\bigcup_{i=0}^{N} V_{b_{i}}$.

The scattering operator $S$ is defined as

$$
S(\vec{r}, t)\left(\vec{k}, b \mid \vec{k}_{0}, b_{0}\right):=w(\vec{r}, t)\left(\vec{k}_{0}, b_{0} \mid \vec{k}, b\right)-\delta^{3}\left(\vec{k}-\vec{k}_{0}\right) \delta_{b, b_{0}} W_{t o t}(\vec{r}, t)(\vec{k}, b),
$$

with $W_{\text {tot }}(\vec{r}, t)(\vec{k}, b):=\sum_{b^{\prime}} \int_{V_{b^{\prime}}} w(\vec{r}, t)\left(\vec{k}, b \mid \vec{k}^{\prime}, b^{\prime}\right) \mathrm{d}^{3} k^{\prime}>0$.

By definition, $w(\vec{r}, t)\left(\vec{k}_{0}, b_{0} \mid \vec{k}, b\right)$ is a bound continuous compact operator on the Banach space $C^{0}(K)$ with $\|\cdot\|_{\infty}$ (see, e.g., $[14$, p. 70$]$ ).

In the remainder, the argument $(\vec{r}, t)$ will be omitted, and the Dirac notation sometimes will be used for better readability (e.g., $|f\rangle:=f$ ). We will also sometimes use the "o" notation

$$
(A \circ B)\left(\vec{k}, b \mid \vec{k}_{1}, b_{1}\right):=\sum_{b_{0}} \int_{V_{b_{0}}} A\left(\vec{k}, b \mid \vec{k}_{0}, b_{0}\right) B\left(\vec{k}_{0}, b_{0} \mid \vec{k}_{1}, b_{1}\right) \mathrm{d}^{3} k_{0}
$$

to avoid confusion. Using the Dirac notation, the lhs of (3.1) can be written as

$$
\begin{aligned}
S|f\rangle:=\sum_{b_{0}} \int_{V_{b_{0}}} S\left(\vec{k}, b \mid \vec{k}_{0}, b_{0}\right) f\left(\vec{k}_{0}, b_{0}\right) \mathrm{d}^{3} k_{0} \\
\quad=\sum_{b_{0}} \int_{V_{b_{0}}}\left(f\left(\vec{k}_{0}, b_{0}\right) w\left(\vec{k}_{0}, b_{0} \mid \vec{k}, b\right)-f(\vec{k}, b) w\left(\vec{k}, b \mid \vec{k}_{0}, b_{0}\right)\right) \mathrm{d}^{3} k_{0} .
\end{aligned}
$$

Now we want to define an inverse operator $H$ for the SO, i.e., an ISO. First of all, one cannot define the ISO naively as $H \circ S|f\rangle=|f\rangle$ for all $f \in C^{0}(K)$, because $S$ has an eigenvector with eigenvalue 0 (indeed only one, as we will show later), namely, the Boltzmann function $f_{e q} .{ }^{1}$ Therefore, we have to invert the SO on the space $K e r^{\perp}$ perpendicular to its kernel Ker $:=\left\{\lambda\left|f_{\text {eq }}\right\rangle \mid \lambda \in \mathbb{R}\right\}$. To do so, we define explicitly

$$
K e r^{\perp}:=\left\{P_{f_{e q}}|g\rangle \mid g \in C^{0}(K)\right\},
$$

\footnotetext{
${ }^{1}$ Also in the case of degenerate systems and/or systems with two-particle scattering (e.g., e-e collisions) the eigenvector with eigenvalue 0 exists and is unique, but it will depend on the solution $f$ of the BE, because the scattering operator $S$ depends on $f$. This will not impact the validity of our approach.
} 
where

$$
P_{f_{e q}}:=\mathbb{1}-\left|f_{e q}\right\rangle\left\langle f_{e q}\right|
$$

$f_{e q}$ is chosen such that

$$
\left\langle f_{e q} \mid f_{e q}\right\rangle=1
$$

and the scalar product is given by

$$
\langle f \mid g\rangle:=\sum_{b} \int_{V_{b}} f(\vec{k}, b) g(\vec{k}, b) \mathrm{d}^{3} k .
$$

A trivial but important property of $S$ is

$$
S|g\rangle=S \circ P_{f_{e q}}|g\rangle .
$$

Now, the ISO $H$, if it exists on $K e r^{\perp}$, can be unequivocally defined on $C^{0}(K)$ based on the properties it must fulfill:

1. $H \circ S|g\rangle \stackrel{!}{=}|g\rangle$ for all $g \in K e r^{\perp}$,

2. $H\left|f_{1}\right\rangle=0$,

with $f_{1}(\vec{k}, b):=\frac{1}{\sqrt{\sum_{b^{\prime}}\left|V_{b^{\prime}}\right|}}=$ const, where $\left|V_{b^{\prime}}\right|$ is the volume of $V_{b^{\prime}}$. Using (3.9), condition 1 can be rewritten as

$$
H \circ S|g\rangle=H \circ S \circ P_{f_{e q}}|g\rangle \stackrel{!}{=} P_{f_{e q}}|g\rangle \forall g \in C^{0}(K) .
$$

The appropriateness of condition 2 will now be explained in detail. First, note that $S^{T}\left|f_{1}\right\rangle=0\left(\left\langle f_{1}\right| S=0\right)$ by definition of $W_{\text {tot }}$. Without condition 2 we could define an infinite number of ISOs, because if $H$ satisfies condition 1, then $H+|v\rangle\left\langle f_{1}\right|$ fulfills the same condition for any $|v\rangle$. Let $H^{*}$ be an ISO fulfilling condition 1 , and $\left|h^{*}\right\rangle:=H^{*}\left|f_{1}\right\rangle$. We can always rewrite $H^{*}$ as $H^{*}=H^{\perp}+\left|h^{*}\right\rangle\left\langle f_{1}\right|$, where $H^{\perp}:=H^{*}-\left|h^{*}\right\rangle\left\langle f_{1}\right|$. (Note here that because $H^{\perp}$ fulfills conditions 1 and 2, it is unambiguously defined and, therefore, independent of $H^{*}$.) By multiplying (3.1) by $\left\langle f_{1}\right|$ we obtain $\partial_{t} n+\nabla_{\mathbf{r}}\langle\dot{\vec{r}}\rangle=0$, which is nothing but the current continuity equation. (In the case of semiconductors, the boundary term $\oint_{\partial V_{b_{0}}} f \dot{\vec{k}} \cdot \vec{n} \mathrm{~d} a$ always disappears due to the inversion symmetry of the $V_{b_{0}}$.) Thus, by multiplying (3.1) by $H^{*}$ and using (3.10), we obtain

$$
\begin{aligned}
H^{*} \partial_{t}|f\rangle+H^{*} \dot{\vec{r}} \cdot \nabla_{\mathbf{r}}|f\rangle+H^{*} \dot{\vec{k}} \cdot \nabla_{\mathbf{k}}|f\rangle & =|f\rangle-\left\langle f \mid f_{e q}\right\rangle\left|f_{e q}\right\rangle \\
& =H^{\perp} \partial_{t}|f\rangle+H^{\perp} \dot{\vec{r}} \cdot \nabla_{\mathbf{r}}|f\rangle+H^{\perp} \dot{\vec{k}} \cdot \nabla_{\mathbf{k}}|f\rangle .
\end{aligned}
$$

The last equation shows that $H^{\perp}$ already contains the full physical information, and that it is reasonable to define $H:=H^{\perp}$, i.e., $H\left|f_{1}\right\rangle=0$.

We can now write an equation for the ISO:

$$
H \circ S|g\rangle \stackrel{!}{=}|g\rangle-\left|f_{e q}\right\rangle\left\langle f_{e q} \mid g\right\rangle \forall g,
$$

and finally write the operator equations for $H$ :

$$
H \circ S \stackrel{!}{=} \delta^{3}\left(\vec{k}-\vec{k}_{0}\right) \delta_{b, b_{0}}-f_{e q}(\vec{k}, b) f_{e q}\left(\vec{k}_{0}, b_{0}\right)=\mathbb{1}-\left|f_{e q}\right\rangle\left\langle f_{e q}\right|,
$$




$$
H\left|f_{1}\right\rangle \stackrel{!}{=} 0 .
$$

Next we have to solve for (3.13), (3.14). From (3.2) and (3.13) we obtain

$$
\begin{array}{r}
H \circ S=\sum_{b_{2}} \int_{V_{b_{2}}} H\left(\vec{k}, b \mid \vec{k}_{2}, b_{2}\right) w\left(\vec{k}_{0}, b_{0} \mid \vec{k}_{2}, b_{2}\right) \mathrm{d}^{3} k_{2}-W_{\text {tot }}\left(\vec{k}_{0}, b_{0}\right) H\left(\vec{k}, b \mid \vec{k}_{0}, b_{0}\right) \\
\stackrel{!}{=} \delta^{3}\left(\vec{k}-\vec{k}_{0}\right) \delta_{b, b_{0}}-f_{\text {eq }}(\vec{k}, b) f_{\text {eq }}\left(\vec{k}_{0}, b_{0}\right) .
\end{array}
$$

By rearranging the terms, this equation can be written as

(3.16) $H\left(\vec{k}, b \mid \vec{k}_{0}, b_{0}\right)$

$=\sum_{b_{2}} \int_{V_{b_{2}}} H\left(\vec{k}, b \mid \vec{k}_{2}, b_{2}\right) \frac{w\left(\vec{k}_{0}, b_{0} \mid \vec{k}_{2}, b_{2}\right)}{W_{\text {tot }}\left(\vec{k}_{0}, b_{0}\right)} \mathrm{d}^{3} k_{2}-\frac{\delta^{3}\left(\vec{k}-\vec{k}_{0}\right) \delta_{b, b_{0}}}{W_{\text {tot }}\left(\vec{k}_{0}, b_{0}\right)}+f_{\text {eq }}(\vec{k}, b) \frac{f_{\text {eq }}\left(\vec{k}_{0}, b_{0}\right)}{W_{\text {tot }}\left(\vec{k}_{0}, b_{0}\right)}$.

Now remember that we are interested only in MISO,

$$
H_{g}\left(\vec{k}_{0}, b_{0}\right):=\sum_{b} \int_{V_{b}} g(\vec{k}, b) H\left(\vec{k}, b \mid \vec{k}_{0}, b_{0}\right) \mathrm{d}^{3} k=\langle g| H,
$$

for which we can rewrite (3.16) as

$$
\begin{aligned}
& \left|H_{g}\right\rangle:=H_{g}\left(\vec{k}_{0}, b_{0}\right) \\
& =\sum_{b_{2}} \int_{V_{b_{2}}} H_{g}\left(\vec{k}_{2}, b_{2}\right) \frac{w\left(\vec{k}_{0}, b_{0} \mid \vec{k}_{2}, b_{2}\right)}{W_{t o t}\left(\vec{k}_{0}, b_{0}\right)} \mathrm{d}^{3} k_{2}-\left(\frac{g\left(\vec{k}_{0}, b_{0}\right)-\langle g\rangle_{e q} f_{e q}\left(\vec{k}_{0}, b_{0}\right)}{W_{t o t}\left(\vec{k}_{0}, b_{0}\right)}\right),
\end{aligned}
$$

where $\langle g\rangle_{e q}:=\left\langle f_{e q} \mid g\right\rangle$.

If we define

$$
\begin{gathered}
A\left(\vec{k}_{0}, b_{0} \mid \vec{k}_{2}, b_{2}\right):=\frac{w\left(\vec{k}_{0}, b_{0} \mid \vec{k}_{2}, b_{2}\right)}{W_{t o t}\left(\vec{k}_{0}, b_{0}\right)}, \\
A^{T}\left(\vec{k}_{0}, b_{0} \mid \vec{k}_{2}, b_{2}\right):=\frac{w\left(\vec{k}_{2}, b_{2} \mid \vec{k}_{0}, b_{0}\right)}{W_{t o t}\left(\vec{k}_{2}, b_{2}\right)}, \\
\left|l_{g}\right\rangle:=l_{g}\left(\vec{k}_{0}, b_{0}\right):=\left(\frac{g\left(\vec{k}_{0}, b_{0}\right)-\langle g\rangle_{e q} f_{e q}\left(\vec{k}_{0}, b_{0}\right)}{W_{t o t}\left(\vec{k}_{0}, b_{0}\right)}\right),
\end{gathered}
$$

we can express (3.18) as

$$
\left|H_{g}\right\rangle=A\left|H_{g}\right\rangle-\left|l_{g}\right\rangle
$$

and (3.14) as

$$
\left\langle H_{g} \mid f_{1}\right\rangle=0
$$

Note that

$$
S\left(\vec{k} \mid \vec{k}_{0}\right)=\left(A^{T}\left(\vec{k} \mid \vec{k}_{0}\right)-\mathbb{1}\right) W_{\text {tot }}\left(\vec{k}_{0}\right) .
$$

So far we found that if, for a given $g$, there exists one and only one solution to (3.20) and (3.21), then this solution will have the properties we are looking for. 
3.2. Computation of the solution. One could think that by iteratively inserting the lhs of (3.20) into the rhs, we could solve the problem, i.e.,

$$
\left|H_{g}\right\rangle=A^{n}\left|H_{g}\right\rangle-\sum_{k=0}^{n-1} A^{k}\left|l_{g}\right\rangle .
$$

However, the way to solve (3.20) is somewhat more involved.

Before we go to the solution we discuss an additional condition we have to impose on $A$. The term $A\left(k, b \mid k_{0}, b_{0}\right)$ is nothing but the probability for a particle to end in $\left(k_{0}, b_{0}\right)$ after one scattering event, having started in $(k, b) . A^{m}\left(k, b \mid k_{0}, b_{0}\right)$ is then the probability of going to $\left(k_{0}, b_{0}\right)$ after $m$ scattering events. In the following we will work under the assumption that there exists an $M \in \mathbb{N}$ such that $A^{M}\left(k, b \mid k_{0}, b_{0}\right)>$ 0 for all $(k, b),\left(k_{0}, b_{0}\right)$. It means that it is possible, starting from any $(k, b)$, to reach any $\left(k_{0}, b_{0}\right)$ after $M$ scattering events. Under this assumption, $A^{M}$ is a strong positive compact operator.

Proposition 3.1. The first Krein-Rutman theorem [9] ensures the existence and uniqueness of a stationary solution in thermodynamic equilibrium $\left(\exists ! f \in C^{0}(K) \mid\right.$ $S|f\rangle=0)$.

Proof. We first prove $r\left(A^{M}\right)=1$. By construction, $\|A\|:=\sup _{x \in C^{0}(K) \backslash\{0\}}$ $\frac{\|A x\|_{\infty}}{\|x\|_{\infty}} \leqslant 1$, and $A\left|f_{1}\right\rangle=\left|f_{1}\right\rangle$. Therefore, $r(A) \geqslant 1$, and because by definition $r(A) \leqslant\|A\|$, we find $\sqrt[M]{r\left(A^{M}\right)}=r(A)=\|A\|=1$. The first Krein-Rutman theorem ensures that there is only one strict positive function $u \in C^{0}(K)$ such that $A^{M} u=$ $r\left(A^{M}\right) u=u$. Of course this function is nothing but $f_{1}$. Now we are interested in $\left(A^{T}\right)^{M}$. By construction, $\left(A^{T}\right)^{M}$ is a strong positive compact operator. Using again the Krein-Rutman theorem we find a unique $v$ with $0<v \in C^{0}(K)$ such that $\left(A^{T}\right)^{M} v=r\left(\left(A^{T}\right)^{M}\right) v$. Because $u>0$ and $v>0$, we find $0<\langle u \mid v\rangle=\left\langle A^{M} u \mid v\right\rangle=$ $\left\langle u \mid\left(A^{T}\right)^{M} v\right\rangle=r\left(\left(A^{T}\right)^{M}\right)\langle u \mid v\rangle$. Thus, $r\left(\left(A^{T}\right)^{M}\right)=1$ and $\left(A^{T}\right)^{M} v=v$. Then, because $\left(A^{T}\right)^{M+1} v=A^{T} v \Longleftrightarrow A^{T} v=c * v$ with $c$ real and $c^{M}=1, A^{T} v=v$. Using (3.22) gives $S f_{e q}=0$ with $f_{e q}:=\frac{v}{W_{t o t}}$. The function $f_{e q}$ is the only solution.

To solve (3.20), we construct a sub-Banach space $Q \subset C^{0}(K)$, where the solution is unique. Using two important properties of $A$,

1. $A\left|f_{1}\right\rangle=\left|f_{1}\right\rangle$ by definition of $W_{t o t}$,

2. $A^{T}\left|W_{\text {tot }} f_{\text {eq }}\right\rangle=\left|W_{\text {tot }} f_{\text {eq }}\right\rangle$ because $S\left|f_{\text {eq }}\right\rangle=0$,

we can define

$$
P_{L}:=\mathbb{1}-\frac{\left|f_{1}\right\rangle\left\langle f_{e q} W_{t o t}\right|}{\left\langle f_{1} \mid f_{e q} W_{t o t}\right\rangle}
$$

which has the following important properties:

$$
\begin{gathered}
P_{L}^{2}=P_{L}, \\
P_{L} \circ A=A \circ P_{L}=P_{L} \circ A \circ P_{L} .
\end{gathered}
$$

Using $P_{L}$, we define the space $Q:=\left\{P_{L} x \mid x \in C^{0}(K)\right\}$. Because $W_{\text {tot }} f_{e q}$ and $f_{1}$ are continuous functions, $Q$ is a Banach space.

Proposition 3.2. A is a linear compact operator on $Q$.

Proof. Let $x \in Q$. By definition of $Q$ and property (3.25), $P_{L} x=x$. Therefore, using property (3.26), $A x=A \circ P_{L} x=P_{L} \circ A x \in Q$. 
Note that $\left|l_{g}\right\rangle \in Q$ because

$$
\left\langle f_{e q} W_{t o t} \mid l_{g}\right\rangle=0 .
$$

By multiplying (3.20) by $P_{L}$ we reformulate the problem on $Q$ :

$$
P_{L}\left|H_{g}\right\rangle=P_{L} \circ A \circ P_{L}\left|H_{g}\right\rangle-\left|l_{g}\right\rangle .
$$

Defining $\left|H_{g}^{\perp}\right\rangle:=P_{L}\left|H_{g}\right\rangle$ we obtain

$$
(\mathbb{1}-A)\left|H_{g}^{\perp}\right\rangle=-\left|l_{g}\right\rangle .
$$

Proposition 3.3. $\left(\mathbb{1}-A^{M}\right)$ is invertible on $Q$, and, therefore, $(\mathbb{1}-A)$ is also invertible on $Q$.

Proof. We first prove $\left\|A^{M}\right\|_{\infty}<1$ on $Q$. Let $x$ be in $Q . \quad x$ is a continuous function and $\left\langle f_{e q} W_{t o t} \mid x\right\rangle=0$ because $\left\langle f_{e q} W_{t o t}\right| P_{L}=0$. By definition, $f_{e q} W_{\text {tot }}$ is a strict positive function, and therefore $x$ must have a positive part and a negative part. We define $x_{+}(k):=x(k)$ if $x(k) \geqslant 0, x_{+}(k)=0$ else, and $x_{-}(k):=x(k)$ if $x(k)<0$, $x_{-}(k)=0$ else. By definition, $x(k)=x_{+}(k)+x_{-}(k)$ for all $k \in K$. Without loss of generality, $\|x\|_{\infty}=\left\|x_{+}\right\|_{\infty}$. Then, remembering that $A^{M}$ is strictly positive, we have $\left|A^{M} x\right|=|| A^{M} x_{+}|-| A^{M} x_{-}||<\left|A^{M} x_{+}\right| \leqslant\left\|x_{+}\right\|_{\infty}$. Therefore, $\left\|A^{M}\right\|_{\infty}<1$. It means that $\left(1-A^{M}\right)$ has an inverse on $Q$ that can be written as a Neumann series: $\left(\mathbb{1}-A^{M}\right)^{-1}=\sum_{i=0}^{\infty} A^{M^{i}}$. Rewriting $\left(\mathbb{1}-A^{M}\right)$ as $(\mathbb{1}-A) \circ \sum_{j=0}^{M-1} A^{j}$ and multiplying by $\left(\mathbb{1}-A^{M}\right)^{-1}$, we find $\mathbb{1}=\sum_{i=0}^{\infty} A^{M^{i}} \circ(\mathbb{1}-A) \circ \sum_{j=0}^{M-1} A^{j}=(\mathbb{1}-A) \circ \sum_{i=0}^{\infty} A^{M^{i}} \circ$ $\sum_{j=0}^{M-1} A^{j}$. Thus, clearly $(\mathbb{1}-A)^{-1}=\sum_{i=0}^{\infty} A^{M^{i}} \circ \sum_{j=0}^{M-1} A^{j}=\sum_{i=0}^{\infty} A^{i}$.

Multiplying (3.29) by $(\mathbb{1}-A)^{-1}$ gives the solution we were looking for:

$$
\left|H_{g}^{\perp}\right\rangle=-\sum_{i=0}^{\infty} A^{i}\left|l_{g}\right\rangle
$$

Thus, problem (3.20) has a unique solution on $Q$, but infinitely many of the form $H_{g}^{\lambda}:=H_{g}^{\perp}+\lambda\left|f_{1}\right\rangle, \lambda \in \mathbb{R}$, on $C^{0}(K)$. In (3.21) we imposed the condition $\left\langle H_{g} \mid f_{1}\right\rangle=0$ to obtain a unique solution. The only $H_{g}^{\lambda}$ fulfilling this condition is $H_{g}^{\lambda_{g}}$ with

$$
\lambda_{g}:=-\left\langle f_{1} \mid H_{g}^{\perp}\right\rangle,
$$

which is the unique solution we are looking for.

Equation (3.30) represents an iterative method for computing $H_{g}^{\perp}$, i.e., for finding an exact solution of (3.13), (3.14) for any $g \in C^{0}(K)$.

As $\left\langle H_{g}\right|=\langle g| H$ exists for all $g \in C^{0}(K), H$ also exists and is unique. Thus, (3.13) together with (3.14) also has a unique solution.

3.3. Connection between $\boldsymbol{H}$ and $\boldsymbol{S}^{-1}$. We show the connection between $H$ and $S^{-1}$, as well as between $H_{g}$ and $S_{g}^{-1}$. If we let the operator $H$ act on both sides of (3.1), we find with (3.13)

$$
H \partial_{t}|f\rangle+H \dot{\vec{r}} \cdot \nabla_{\mathbf{r}}|f\rangle+H \dot{\vec{k}} \cdot \nabla_{\mathbf{k}}|f\rangle=|f\rangle-\left\langle f \mid f_{e q}\right\rangle\left|f_{e q}\right\rangle .
$$

We want to dispose of the term $\left\langle f \mid f_{e q}\right\rangle$ and replace it with a term containing the density. By computing the 0th moment of (3.32), we obtain

$$
\begin{aligned}
& \left\langle H_{1}\left|\partial_{t}\right| f\right\rangle+\left\langle H_{1}\left|\dot{\vec{r}} \cdot \nabla_{\mathbf{r}}\right| f\right\rangle+\left\langle H_{1}\left|\dot{\vec{k}} \cdot \nabla_{\mathbf{k}}\right| f\right\rangle=n-\left\langle f \mid f_{e q}\right\rangle n_{e q} \\
& \Leftrightarrow\left\langle f \mid f_{e q}\right\rangle=\frac{n}{n_{e q}}-\frac{1}{n_{e q}}\left\langle H_{1}\left|\partial_{t}\right| f\right\rangle-\frac{1}{n_{e q}}\left\langle H_{1}\left|\dot{\vec{r}} \cdot \nabla_{\mathbf{r}}\right| f\right\rangle-\frac{1}{n_{e q}}\left\langle H_{1}\left|\dot{\vec{k}} \cdot \nabla_{\mathbf{k}}\right| f\right\rangle,
\end{aligned}
$$


where $H_{1}\left(\vec{k}_{0}, b_{0}\right):=\sum_{b} \int_{V_{b}} H\left(\vec{k}, b \mid \vec{k}_{0}, b_{0}\right) \mathrm{d}^{3} k$ and $n_{e q}:=\sum_{b} \int_{V_{b}} f_{e q}(\vec{k}, b) \mathrm{d}^{3} k$. Inserting (3.33) into (3.32) results in

$$
\begin{gathered}
\left(H-\frac{\left|f_{e q}\right\rangle\left\langle H_{1}\right|}{n_{e q}}\right) \partial_{t}|f\rangle+\left(H-\frac{\left|f_{e q}\right\rangle\left\langle H_{1}\right|}{n_{e q}}\right) \dot{\vec{r}} \cdot \nabla_{\mathbf{r}}|f\rangle+\left(H-\frac{\left|f_{e q}\right\rangle\left\langle H_{1}\right|}{n_{e q}}\right) \dot{\vec{k}} \cdot \nabla_{\mathbf{k}}|f\rangle \\
=|f\rangle-\left|f_{e q}\right\rangle \frac{n}{n_{e q}}
\end{gathered}
$$

With the definition $S^{-1}\left(\vec{k}, b \mid \vec{k}_{0}, b_{0}\right):=H\left(\vec{k}, b \mid \vec{k}_{0}, b_{0}\right)-f_{e q}(\vec{k}, b) H_{1}\left(\vec{k}_{0}, b_{0}\right) / n_{e q}$ we find (2.4). Finally, one obtains for the $g$-moment

$$
S_{g}^{-1}\left(\vec{k}_{0}, b_{0}\right):=H_{g}\left(\vec{k}_{0}, b_{0}\right)-\frac{\langle g\rangle_{e q} H_{1}\left(\vec{k}_{0}, b_{0}\right)}{n_{e q}} .
$$

Note that $S_{g}^{-1}\left(\vec{k}_{0}, b_{0}\right)$ fulfills by definition the equations

$$
\begin{gathered}
A S_{g}^{-1}=S_{g}^{-1}(\vec{k}, b)+\left(\frac{g(\vec{k}, b)-\frac{\langle g\rangle_{e q}}{n_{e q}}}{W_{t o t}(\vec{k}, b)}\right) \Leftrightarrow\left|S_{g}^{-1}\right\rangle=A\left|S_{g}^{-1}\right\rangle-\left|h_{g}\right\rangle, \\
\left\langle S_{g}^{-1} \mid f_{1}\right\rangle=0,
\end{gathered}
$$

where $h_{g}(\vec{k}, b):=\left(g(\vec{k}, b)-\frac{\langle g\rangle_{e q}}{n_{e q}}\right) / W_{t o t}(\vec{k}, b)$ (compare with (3.20) and (3.21)).

The proof of the existence and of the uniqueness of $S_{g}^{-1}$ up to a constant is herewith completed.

Putting (3.30) and (3.35) together leads to

$$
\left|S_{g}^{-1}\right\rangle=-\left(\mathbb{1}-\left|f_{1}\right\rangle\left\langle f_{1}\right|\right) \circ \sum_{i=0}^{\infty}\left(P_{L} \circ A \circ P_{L}\right)^{i}\left|h_{g}\right\rangle=-\left(\mathbb{1}-\left|f_{1}\right\rangle\left\langle f_{1}\right|\right) \circ \sum_{i=0}^{\infty} A^{i}\left|h_{g}\right\rangle,
$$

which is an iterative method for computing $S_{g}^{-1}$ for any given operator $S$ and function $g$.

3.4. The Dirac delta distribution. Before going to the applications we would like to discuss the hypothesis on $w\left(\vec{k}, b \mid \vec{k}^{\prime}, b^{\prime}\right)$. At the beginning of this section we chose $w\left(\vec{k}, b \mid \overrightarrow{k^{\prime}}, b^{\prime}\right)$ to be a continuous function of its arguments $\vec{k}$ and $\vec{k}^{\prime}$. In many "physical" models, however, the function $w\left(\vec{k}, b \mid \overrightarrow{k^{\prime}}, b^{\prime}\right)$ is replaced with a distribution (usually a sum of Dirac's delta "functions" of a continuous function of $\vec{k}$ and $\vec{k}^{\prime}$ ). In the following we argue against delta distributions (functions) based on the concept of regularization of the delta distribution. A function belonging to a family of continuous functions $\delta_{\gamma}(\varepsilon)$ depending on a continuous parameter $\gamma$ is called a regularization of the delta function iff $\lim _{\gamma \rightarrow \infty} \int_{-\infty}^{\infty} \delta_{\gamma}(\varepsilon) f(\varepsilon) d \varepsilon=f(0)$ for all continuous functions of the energy $f(\varepsilon)$, and $\int_{-\infty}^{\infty} \delta_{\gamma}(\varepsilon) d \varepsilon=1$ for all $\gamma$. Now, remember that the delta distributions contained in the usual scattering rates arise from Fermi's golden rule, i.e., from the regularization $\delta_{\gamma}^{(F)}(\varepsilon):=\left(\sin (\varepsilon \gamma)^{2}\right) /\left(\gamma \pi \varepsilon^{2}\right)$. Replacing in the scattering rate $w$ the delta distributions with $\delta_{\gamma}^{(F)}(\varepsilon)$ for any $\gamma<\infty$, the hypotheses on $w$ are again fulfilled. This fact can be interpreted physically, mathematically, and numerically. 
Physically, a simple argument can be found for why $\gamma$ should be smaller than $\infty$ : From the Heisenberg uncertainty principle there is no exact energy conservation in a finite amount of time. Thus, the delta distribution is just a useful approximation. Mathematically, the situation is quite different if one considers the limit $\gamma \rightarrow \infty$ before or after computing the MISO. Taking the limit before the computation of the MISO leads to an operator $A$, which is not compact and, even worse, leads to infinitely many solutions to the BE at thermodynamic equilibrium (see [11]). Taking the limit after the computation of the MISO trivially leads to a single well-defined solution for each $g \in C^{0}(K)$. Numerically, a computer using double arithmetic cannot digitize the difference between a delta distribution and, e.g., the regularization $\delta_{\gamma}^{(a)}(\varepsilon)$ such that $\operatorname{supp}\left(\delta_{\gamma}^{(a)}\right)=\left[-\gamma^{-1}, \gamma^{-1}\right]$ and $\gamma>10^{307}$. This means that when solving the $\mathrm{BE}$ on a computer, one is actually working with a delta distribution. For these reasons we conclude that a delta distribution contained in the scattering rates can and should be replaced with a well-chosen regularization. Metaphorically speaking, delta distributions give birth to operators which are a bit like monsters (due to their noncompactness) and, although they are interesting objects from a mathematical point of view (see, e.g., [2] and [11]), they create artificial problems from a physical point of view.

\section{Applications.}

4.1. Introduction. The knowledge of the MISO and of the solution $f$ of the $\mathrm{BE}$ is necessary and sufficient to compute all transport parameters next to and far from thermodynamic equilibrium. In this section, five important applications are presented.

4.2. Low-field solution to the BE. The space-homogeneous, stationary BE

$$
-\frac{q}{\hbar} \vec{E} \cdot \nabla_{\mathbf{k}}|f\rangle-q(\vec{v} \wedge \vec{B}) \cdot \nabla_{\mathbf{k}}|f\rangle=S|f\rangle
$$

can be solved for small electric and magnetic fields using the ansatz (see, e.g., [10])

$$
f(\vec{k})=f_{e q}(\varepsilon(\vec{k}))+q \frac{\partial f_{e q}}{\partial \varepsilon}(\varepsilon(\vec{k})) \vec{E} \cdot \vec{\Lambda}^{a}(\vec{k})+q \frac{\partial f_{e q}}{\partial \varepsilon}(\varepsilon(\vec{k}))(\vec{v} \wedge \vec{B}) \cdot \vec{\Lambda}^{b}(\vec{k}) .
$$

Inserting (4.2) into (4.1) and taking into account only the first order terms in the magnetic and electric fields leads to

$$
(\vec{v} \wedge \vec{B}) \cdot \vec{\Lambda}^{b}=0
$$

because $(\vec{v} \wedge \vec{B}) \cdot \vec{v}=0$, and

$$
\left\langle\left(1-f_{e q}\right)(\vec{v})_{i}\left|=-\left\langle\left(\vec{\Lambda}^{a}\right)_{i}\right)\right| S .\right.
$$

It is important that (4.4) be derived only by using the principle of detailed balance. The solution to (4.4) is trivially

$$
\left(\vec{\Lambda}^{a}\right)_{i}(\vec{k})=-S_{\left(1-f_{e q}\right) v_{i}}^{-1}(\vec{k}) .
$$

Therefore, the solution to the low-field BE is

$$
f(\vec{k})=f_{e q}\left(1+\frac{q}{k_{B} T}\left(1-f_{e q}\right) \vec{E} \cdot S_{\left(1-f_{e q}\right) \vec{v}}^{-1}\right)
$$


in the case of Fermi-Dirac statistics, and

$$
f(\vec{k})=f_{e q}\left(1+\frac{q}{k_{B} T} \vec{E} \cdot S_{\vec{v}}^{-1}\right)
$$

in the case of Boltzmann statistics.

In the low-field case the solution of the BE is therefore determined only by the equilibrium distribution $f_{e q}$ and by $S_{\left(1-f_{e q}\right) \vec{v}}^{-1}\left(\right.$ resp., $\left.S_{\vec{v}}^{-1}\right)$.

4.3. Transport parameters. As already mentioned in section 2 , tensorial transport parameters can be exactly computed using MISO. For example, the mobility is given by

$$
\mu_{i j}:=\frac{q}{n \hbar} \int_{K} S_{v_{i}}^{-1} \partial_{k_{j}} f d^{3} k
$$

and the diffusivity tensor by

$$
D_{i j}:=-\frac{1}{n} \int_{K} S_{v_{i}}^{-1} v_{j} f d^{3} k .
$$

Note that in the case of Boltzmann statistics, setting $f=f_{e q}$ in (4.8) and (4.9) yields the well-known Einstein relation for all components of the tensors

$$
\frac{k_{B} T}{q} \mu_{i j}=D_{i j}
$$

These transport coefficients are exact and unique. Their definition does not require any restrictions except those already contained in the BE. If they are used in the associated TM, its solution will reproduce the corresponding moment(s) of the BE. In two and three dimensions this is, to the authors' knowledge, the first generally valid scheme ever described which can be used to compute tensorial transport coefficients for all possible geometries and configurations.

A straightforward application is the customization of the model for the transport coefficients for a given device. Using (4.8) and (4.9), transport coefficients can be computed in a device for different bias points (using, e.g., the MC method), especially in the parts of the device where the usual bulk models for the transport coefficients are no longer valid. Then, a customized model for the device can be extracted by choosing a proper local parametrization. In the case of the DD model the transport coefficients can be parametrized using, e.g., the local electric field or the local current density. This custom model will be, of course, valid only for the considered device, but nevertheless, it will enable us to compute the direct current (DC), alternating current (AC), and noise characteristics of the device in a much simpler way than by directly working with the BE.

4.4. Hall factor. When a constant voltage is applied between $A$ and $B$ (see Figure 4.1), and a constant magnetic field $B_{z^{\prime}}$ is present in the $z^{\prime}$ direction, then two Hall factors can be defined,

$$
\begin{aligned}
R_{H} & :=\frac{V_{21}}{d_{1} J_{x^{\prime}} B_{z^{\prime}}}, \\
R_{H}^{*} & :=\frac{V_{43}}{d_{2} J_{x^{\prime}} B_{z^{\prime}}},
\end{aligned}
$$




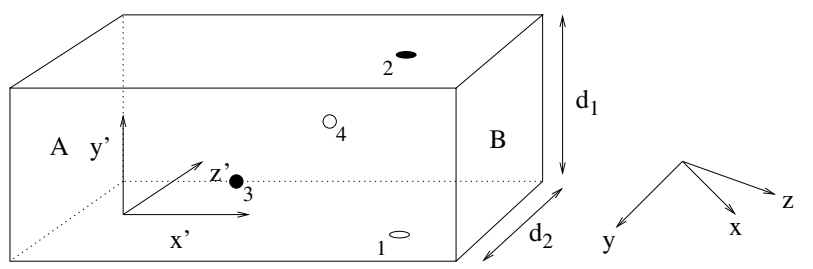

FIG. 4.1. Piece of bulk material.

where $V_{21}$ is the voltage between the points 1 and $2, V_{43}$ is the voltage between the points 3 and 4 , and $J_{x^{\prime}}$ is the current density in $x^{\prime}$-direction. Under the assumption that the electric fields $E_{y^{\prime}}$ in the $y^{\prime}$-direction and $E_{z^{\prime}}$ in the $z^{\prime}$-direction are constant, the definitions can be rewritten as

$$
\begin{aligned}
R_{H} & :=\frac{E_{y^{\prime}}}{J_{x^{\prime}} B_{z^{\prime}}}, \\
R_{H}^{*} & :=\frac{E_{z^{\prime}}}{J_{x^{\prime}} B_{z^{\prime}}} .
\end{aligned}
$$

To obtain expressions for $R_{H}$ and $R_{H}^{*}$, the current density equation in the spacehomogeneous case can be written as

$$
\frac{\vec{J}}{n q}=\mu \vec{E}+\alpha \vec{B}
$$

where $\mu$ is defined in (4.8), and $\alpha$ is defined as

$$
\alpha_{i j}:=\frac{1}{n} \int_{K} S_{v_{i}}^{-1}\left(\vec{v} \wedge \nabla_{\mathbf{k}} f\right)_{j} d^{3} k .
$$

For better readability, only the case of Boltzmann statistics will be considered.

In the low-field case, $f$ can be replaced with (4.7), leading to

$$
\alpha_{i j}=\frac{1}{n} \frac{q}{k_{B} T} \int_{K} S_{v_{i}}^{-1}\left(\vec{v} \wedge \nabla_{\mathbf{k}}\left(f_{e q} \vec{E} S_{\vec{v}}^{-1}\right)\right)_{j} d^{3} k,
$$

where the field-independent term disappeared because $\vec{v} \wedge \vec{v}=0$. Taking advantage of the linearity of (4.17) in $\vec{E},(4.15)$ can be rewritten as

$$
\frac{\vec{J}}{n q}=\mu \vec{E}+B_{x} \gamma_{x} \vec{E}+B_{y} \gamma_{y} \vec{E}+B_{z} \gamma_{z} \vec{E},
$$

with

$$
\left(\gamma_{l}\right)_{i j}:=\frac{1}{n} \frac{q}{k_{B} T} \int_{K} S_{v_{i}}^{-1}\left(\vec{v} \wedge \nabla_{\mathbf{k}}\left(f_{e q} S_{v_{j}}^{-1}\right)\right)_{l} d^{3} k .
$$

Therefore,

$$
\vec{E}=\left(\mu+B_{x} \gamma_{x}+B_{y} \gamma_{y}+B_{z} \gamma_{z}\right)^{-1} \frac{\vec{J}}{n q}
$$


If $R$ is the matrix that transforms the $(x, y, z)$-coordinate system into the $\left(x^{\prime}, y^{\prime}, z^{\prime}\right)$ coordinate system, then the Hall factors can be written as

$$
\begin{aligned}
R_{H} & =\frac{\left(R\left(\mu+B_{x} \gamma_{x}+B_{y} \gamma_{y}+B_{z} \gamma_{z}\right)^{-1} R^{-1}\right)_{y x}}{q n B_{z^{\prime}}}, \\
R_{H}^{*} & =\frac{\left(R\left(\mu+B_{x} \gamma_{x}+B_{y} \gamma_{y}+B_{z} \gamma_{z}\right)^{-1} R^{-1}\right)_{z x}}{q n B_{z^{\prime}}} .
\end{aligned}
$$

Equation (4.21) is more general than the formula given in [7] and reduces to the formula given in [10] in special cases. In the case of unstrained bulk silicon, e.g., because of the symmetries of the crystal, (4.18) takes the form

$$
\frac{\vec{J}}{n_{e q} q}=\mu_{e q} \vec{E}-\gamma_{e q} \vec{B} \wedge \vec{E},
$$

where

$$
\begin{gathered}
\mu_{e q}:=\frac{q}{n_{e q} \hbar} \int_{K} S_{v_{x}}^{-1} \partial_{k_{x}} f_{e q} d^{3} k \\
\gamma_{e q}:=\frac{1}{n_{e q}} \frac{q}{k_{B} T} \int_{K} S_{v_{x}}^{-1}\left(\vec{v} \wedge \nabla_{\mathbf{k}}\left(f_{e q} S_{v_{y}}^{-1}\right)\right)_{z} d^{3} k .
\end{gathered}
$$

The Hall factors are then

$$
\begin{gathered}
R_{H}=\frac{1}{q n_{e q}} \frac{\gamma_{e q}}{\mu_{e q}^{2}+\gamma_{e q}^{2} B_{z^{\prime}}^{2}}, \\
R_{H}^{*}=0
\end{gathered}
$$

where $R_{H}$ and $R_{H}^{*}$ are independent of the transformation matrix $R$, i.e., of the crystal orientation.

4.5. RTs. The RT for the $g$-moments of the space-homogeneous BE is usually computed using the formula (see $[8$, p. 136])

$$
\tau_{g}=-\frac{\left\langle g \mid f-f_{e q}\right\rangle}{\langle g|S| f\rangle} .
$$

At least in all semiconductors (strained and unstrained), (4.28) is fully inappropriate, because in the low-field limit (4.28) reduces to the singular expression $\frac{0}{0}$ in the case of even functions $g$, such as, e.g., $\varepsilon, \varepsilon^{2}, v^{2}$, and $v^{4}$. To solve this problem, the alternative expression

$$
\tau_{g}=-\frac{\vec{n} \cdot \int\left(\nabla_{\mathbf{k}} f\right) S_{g}^{-1} d^{3} k}{\vec{n} \cdot \int\left(\nabla_{\mathbf{k}} f\right) g d^{3} k}
$$

can be used, where $\vec{n}$ is the vector pointing in the direction of the electric field. Equation (4.29) never becomes singular in the limit of vanishingly small electric fields.

An extensive application of this theory to silicon can be found in [4]. 
4.6. Langevin noise sources. We are interested in the BE with an additional Langevin term, the so-called "Boltzmann-Langevin" equation (BLE)

$$
\partial_{t} f(\vec{r}, t, \vec{k}, b)+\dot{\vec{r}} \cdot \nabla_{\mathbf{r}} f(\vec{r}, t, \vec{k}, b)+\dot{\vec{k}} \cdot \nabla_{\mathbf{k}} f(\vec{r}, t, \vec{k}, b)=S f+\delta s(\vec{r}, t, \vec{k}, b) .
$$

The BE describes the average state of an infinite number of systems with identical initial conditions, whereas the BLE describes the evolution of one of these systems. The Langevin source term is responsible for the deviation from the average state.

By multiplying (4.30) by $S_{g}^{-1}$, we obtain

$$
\left\langle S_{g}^{-1} \mid \partial_{t} f\right\rangle+\left\langle S_{g}^{-1} \mid \dot{\vec{r}} \cdot \nabla_{\mathbf{r}} f\right\rangle+\left\langle S_{g}^{-1} \mid \dot{\vec{k}} \cdot \nabla_{\mathbf{k}} f\right\rangle=\langle g\rangle-\langle g\rangle_{e q} \frac{n}{n_{e q}}+\left\langle S_{g}^{-1} \mid \delta s\right\rangle .
$$

We want to derive an expression for the Fourier transform of correlation functions of $\left\langle S_{g}^{-1} \mid \delta s\right\rangle$ around a stationary state for the homogeneous BLE with constant density $n$; i.e., we want to compute

$C_{g g^{\prime}}(\omega):=\int_{-\infty}^{\infty} \lim _{T \rightarrow \infty} \frac{1}{2 T} \int_{-T}^{T}\left(\left\langle S_{g}^{-1} \mid \delta s\right\rangle\right)(t)\left(\left\langle S_{g^{\prime}}^{-1} \mid \delta s\right\rangle\right)(t+s) d t e^{-i \omega s} d s$ for constant $n$.

We define the correlation function

$$
C(\omega)\left(\vec{k}, b \mid \vec{k}_{0}, b_{0}\right):=\int_{-\infty}^{\infty} \lim _{T \rightarrow \infty} \frac{1}{2 T} \int_{-T}^{T} \delta s(t)(\vec{k}, b) \delta s(t+s)\left(\vec{k}_{0}, b_{0}\right) d t e^{-i \omega s} d s .
$$

Note that

$$
C_{g g^{\prime}}(\omega)=\sum_{b, b_{0}} \int_{V_{b}} \int_{V_{b_{0}}} S_{g}^{-1}(\vec{k}, b) S_{g^{\prime}}^{-1}\left(\vec{k}_{0}, b_{0}\right) C(\omega)\left(\vec{k}, b \mid \vec{k}_{0}, b_{0}\right) \mathrm{d}^{3} k \mathrm{~d}^{3} k_{0} .
$$

We know from [5, eq. (1.55a), p. 21], that for a homogeneous nondegenerate system with given density, we obtain

$$
\begin{aligned}
& C(\omega)\left(\vec{k}, b \mid \vec{k}_{0}, b_{0}\right) \\
& =\delta^{3}\left(\vec{k}-\vec{k}_{0}\right) \delta_{b, b_{0}} \sum_{b_{1}} \int_{V_{b_{1}}} w\left(\vec{k}, b \mid \vec{k}_{1}, b_{1}\right) f(\vec{k}, b) \mathrm{d}^{3} k_{1} \\
& +\delta^{3}\left(\vec{k}-\vec{k}_{0}\right) \delta_{b, b_{0}} \sum_{b_{1}} \int_{V_{b_{1}}} w\left(\vec{k}_{1}, b_{1} \mid \vec{k}, b\right) f\left(\vec{k}_{1}, b_{1}\right) \mathrm{d}^{3} k_{1} \\
& \quad-w\left(\vec{k}_{0}, b_{0} \mid \vec{k}, b\right) f\left(\vec{k}_{0}, b_{0}\right)-w\left(\vec{k}, b \mid \vec{k}_{0}, b_{0}\right) f(\vec{k}, b),
\end{aligned}
$$

where $f(\vec{k}, b)$ is the stationary homogeneous solution to (3.1). ${ }^{2}$

By plugging (4.35) into (4.34) and rearranging terms, we obtain

$$
C_{g g^{\prime}}(\omega)=\sum_{b} \int_{V_{b}} K_{g g^{\prime}}(\vec{k}, b) f(\vec{k}, b) \mathrm{d}^{3} k=\left\langle K_{g g^{\prime}}\right\rangle,
$$

\footnotetext{
${ }^{2}$ In the case of particle-particle scattering the corresponding additional contribution to the correlation function has to be added (see [5, eq. (1.55b), p. 21]).
} 
where

$$
K_{g g^{\prime}}(\vec{k}, b):=\sum_{b_{0}} \int_{V_{b_{0}}} w\left(\vec{k}, b \mid \vec{k}_{0}, b_{0}\right)\left[\begin{array}{l}
S_{g}^{-1}(\vec{k}, b) S_{g^{\prime}}^{-1}(\vec{k}, b) \\
-S_{g}^{-1}(\vec{k}, b) S_{g^{\prime}}^{-1}\left(\vec{k}_{0}, b_{0}\right) \\
-S_{g}^{-1}\left(\vec{k}_{0}, b_{0}\right) S_{g^{\prime}}^{-1}(\vec{k}, b) \\
+S_{g}^{-1}\left(\vec{k}_{0}, b_{0}\right) S_{g^{\prime}}^{-1}\left(\vec{k}_{0}, b_{0}\right)
\end{array}\right] \mathrm{d}^{3} k_{0} .
$$

Note that (4.37) is invariant under the transformation $S_{g}^{-1}(\vec{k}, b) \rightarrow S_{g}^{-1}(\vec{k}, b)+$ $\alpha_{g} f_{1}$. Therefore, $C_{g g^{\prime}}(\omega)$ is independent of condition (3.14), as it should be.

Thus, the function $C_{g g^{\prime}}(\omega)$ is nothing but the expectancy of $K_{g g^{\prime}}$. Since we can compute $K_{g g^{\prime}}$, we can also compute $\left\langle K_{g g^{\prime}}\right\rangle$ in a very efficient way with an MC simulation. We call $C_{g g^{\prime}}(\omega)$ the Langevin noise source of the functions $g, g^{\prime}$. It describes white noise because it does not depend on $\omega$.

5. Conclusion. The formalism developed in section 3 cannot only be used for studying interesting systems like strained semiconductors, where the SO is fully dependent on the band-valley index, but also for studying electron-hole systems. To do so we have only to formally replace the distribution function $f_{h}$ of the holes in the valence bands by $f_{e}:=1-f_{h}$, i.e., the distribution function for the electrons in the valence bands.

We have described a method based on exact $S_{g}^{-1}$ moments of the inverse scattering operator (MISO) of the Boltzmann equation (BE). This formalism is therefore free of any relaxation time approximation (RTA). We have shown under what sufficient conditions the $S_{g}^{-1}$ exist, and we gave an explicit algorithm to compute them.

We have demonstrated that the knowledge of the $S_{g}^{-1}$ enables the exact computation of transport parameters, correlation functions, and Langevin noise sources. Moreover, the important assumptions underlying the transport models (TMs) and method such as, e.g., the impedance field method (IFM) can be critically examined by our approach.

In forthcoming papers, we will give a general discretization scheme to numerically compute any MISO and extend the method to time-dependent scattering operators (SOs).

Acknowledgments. The authors would like to thank Dr. F. Geelhaar, Dr. B. Schmithüsen, Priv.-Doz. Dr. F. M. Bufler, and T. Bühler of the ETH Zurich for helpful discussions.

\section{REFERENCES}

[1] A. M. Anile, V. Romano, and G. Russo, Extended hydrodynamical model of carrier transport in semiconductors, SIAM J. Appl. Math., 61 (2000), pp. 74-101.

[2] J. BANASIAK, On well-posedness of a Boltzmann-like semiconductor model, Math. Models Methods Appl. Sci., 13 (2003), pp. 875-892.

[3] K. BLøTEKJÆR, Transport equations for electrons in two-valley semiconductors, IEEE Trans. Electron Devices, ED-17 (1970), pp. 38-47.

[4] S. C. BRUgGer AND A. SChenk, First-principle computation of relaxation times in semiconductors for low and high electric fields, in Proceedings of the International Conference on Simulation of Semiconductor Processes and Devices (SISPAD), IEEE, Piscataway, NJ, 2005, pp. 151-154.

[5] S. V. Gantsevich, V. L. Gurevich, and R. Katilius, Theory of fluctuations in nonequilibrium electron gas, Riv. Nuovo Cimento (3), 2 (1979), pp. 1-87.

[6] T. Grasser, H. Kosina, M. Gritsch, And S. SelberherR, Using six moments of Boltzmann's transport equation for device simulation, J. Appl. Phys., 90 (2001), pp. 2389-2396. 
[7] C. Jungemann, M. Bartels, S. Keith, and B. Meinerzhagen, Efficient methods for Hall factor and transport coefficient evaluation for electrons and holes in Si and SiGe based on a full-band structure, in Extended Abstracts of the Sixth International Workshop on Computational Electronics, IEEE, Piscataway, NJ, 1998, pp. 104-107.

[8] C. Jungemann and B. Meinerzhagen, Hierarchical Device Simulation. The Monte-Carlo Perspective, Computational Microelectronics, Springer, Vienna, New York, 2003.

[9] M. G. Krein and M. A. Rutman, Linear operators leaving invariant a cone in a Banach space, Uspehi Matem. Nauk (N.S.), 3 (1948), pp. 3-95 (in Russian); Amer. Math. Soc. Translation, 1950, No. 26 (in English).

[10] O. Madelung, Introduction to Solid-State Theory, Solid-State Sciences, Springer, Berlin, Heidelberg, New York, 1996.

[11] A. MajorAna, Space homogeneous solutions of the Boltzmann equation describing electronphonon interactions in semiconductors, Transport Theory Statist. Phys., 20 (1991), pp. 261-279.

[12] A. Schenk, Advanced Physical Models for Silicon Device Simulation, Computational Microelectronics, Springer, Vienna, New York, 1998.

[13] R. Stratton, Diffusion of hot and cold electrons in semiconductor barriers, Phys. Rev. 126 (1962), pp. 2002-2014.

[14] D. Werner, Funktionalanalysis, 3rd ed., Springer-Verlag, Berlin, 2000. 